\title{
Strengthened ordered directional and other generalizations of monotonicity for aggregation functions
}

\author{
Mikel Sesma-Sara ${ }^{1}$, Laura De Miguel ${ }^{1}$, Julio Lafuente $^{2}$, Edurne Barrenechea $^{1}$, \\ Radko Mesiar ${ }^{3,4}$, and Humberto Bustince ${ }^{1}$ \\ 1 Departamento de Automatica y Computacion and Institute of Smart Cities, \\ Universidad Publica de Navarra, Campus Arrosadia s/n, 31006 Pamplona, Spain \\ \{mikel. sesma, laura.demiguel, edurne. barrenechea, bustince\}@unavarra.es \\ 2 Departamento de Matematicas, Universidad Publica de Navarra, Campus \\ Arrosadia s/n, 31006 Pamplona, Spain \\ lafuente@unavarra.es \\ 3 Department of Mathematics and Descriptive Geometry, Faculty of Civil \\ Engineering, Slovak University of Technology, Radlinského 11, Bratislava, Slovakia \\ mesiar@math.sk \\ 4 Institute for Research and Applications of Fuzzy Modelling, University of Ostrava, \\ 30. dubna 22, Ostrava 1, Czech Republic
}

\begin{abstract}
A tendency in the theory of aggregation functions is the generalization of the monotonicity condition. In this work, we examine the latest developments in terms of different generalizations. In particular, we discuss strengthened ordered directional monotonicity, its relation to other types of monotonicity, such as directional and ordered directional monotonicity and the main properties of the class of functions that are strengthened ordered directionally monotone. We also study some construction methods for such functions and provide a characterization of usual monotonicity in terms of these notions of monotonicity.
\end{abstract}

Keywords: Aggregation functions, directional monotonicity, generalizations of monotonicity, strengthened ordered directional monotonicity

\section{Introduction}

The problem of finding a single representative number for a set of values is common to every field that handles real data. There exist several works in the literature addressing this issue prior to the introduction of the theory of aggregation functions per se. That is the case, for example, of triangular norms [13], copulas [17 and Choquet integrals [7]. According to [14, the inception of the theory of aggregation functions as an independent theory dates back to 1988 [10] and it was not until 2001 that the first monograph on the subject came out [6]. An aggregation function, in the classical sense, is a function $A:[0,1]^{n} \rightarrow[0,1]$ such that $A(\mathbf{0})=0, A(\mathbf{1})=1$ and it is increasing with respect to every argument (the standard partial order on $\left.[0,1]^{n}\right)$. Since its appearence, the aggregation theory 
has been extended to new domains beside real numbers [18 and aggregation functions have been applied in diverse real world problems [8]15].

In addition to the extension of aggregation operators to be able to deal with more general scales than numbers, such as lattices, a relevant trend in the theory of aggregation functions is the relaxation of the monotonicity condition. Monotonicity with respect to every argument may lead to exclude from the framework of aggregation functions mappings that are valid to provide a representative value from a set of numerical values. Examples of such functions are the mode operator, or the Lehmer mean [3], among others (see [2]).

On the account of broadening the framework of functions that are sound for fusing data, some generalizations of monotonicity have emerged [1. One of the most significant forms of monotonicity is that of directional monotonicity, introduced in [5], which, similar to the concept of directional derivatives, deals with monotonicity along a ray in $\mathbb{R}^{n}$. This type of monotonicity generalizes the formerly presented notion of weak monotonicity [19, which is the particular case of restricting directional monotonicity to the ray $(1,1, \ldots, 1)$. The fact that the ray of increasingness could be any vector in $\mathbb{R}^{n}$ permits to select functions that adjust better to particular problems or applications. Nevertheless, that direction is the same for all the points in the domain.

Recently, influenced by the concept of OWA operator 20, the concept of ordered directional (OD) monotonicity has been introduced 4]. The direction of increasingness or decreasingness for ordered directionally monotone function varies depending on the point of the domain that is being considered. Specifically, the ray of increasingness (decreasingness) varies according to the relative size of the inputs, as long as a fixed comonotonicity requirement is satisfied. Directionally and ordered directionally monotone functions have yielded good results in classification problems [12] and in the field of image processing [16].

In this work, we discuss the notion of strengthened ordered directional (SOD) monotonicity [11, a concept based on ordered directional monotonicity, for whose definition no comonotonicity condition is required. This relaxation makes the family of strengthened ordered directionally monotone functions a proper subset of the class of ordered directionally monotone functions, meaning that if a function is strengthened ordered directionally monotone, then it is ordered directionally monotone, but not contrarily. Furthermore, we address some relevant properties of the three forms of monotonicity, i.e., directional, ordered directional and strengthened ordered directional monotonicity, and we point out some links and differences among them. We also expose some construction methods for functions that are monotone in each of the discussed senses and we characterize classical monotonicity with regard to its various generalizations.

This work is organized as follows. We start the next section with some remarks about the notation that is used throughout the paper, as well as recalling some preliminary notions and basic definitions. In Section 3 we present the definition of the latest generalization of monotonicity in the literature regarding directional monotonicity; the concept of strengthened ordered directional monotonicity. We also study the class of SOD functions and we expose a scheme of the 
points that trivially satisfy the conditions for each of the types of monotonicity. In Section 4 we discuss some relevant properties of the different notions of monotonicity, as well as the relations that exist among them. In Section 5 we present various construction methods for functions that are monotone in each sense. We finish this work with a characterization of the usual condition of monotonicity in terms of the different generalizations, in Section 5, followed by some brief concluding remarks.

\section{Preliminaries}

Let $n \in \mathbb{N}$, with $n \geq 2$. We refer as $\mathbf{x}=\left(x_{1}, \ldots, x_{n}\right) \in[0,1]^{n}$ to points in the unit hypercube and as $\vec{r}=\left(r_{1}, \ldots, r_{n}\right) \in \mathbb{R}^{n}$ to vectors connoting a direction in $\mathbb{R}^{n}$.

The notion of monotonicity is highly related to the concept of order. In this work we consider the usual partial order of $[0,1]^{n}$, i.e., given $\mathbf{x}, \mathbf{y} \in[0,1]^{n}$,we say $\mathbf{x} \leq \mathbf{y}$ if $x_{i} \leq y_{i}$ for every $i \in\{1, \ldots, n\}$.

Like in the case of OD monotone functions, the points of the domain whose components are decreasingly ordered play an important role in the framework of SOD monotone functions. We use the following notation for the set of these points: Let $H \subset \mathbb{R}^{n}$, then we set $H_{(\geq)}=\left\{\left(h_{1}, \ldots, h_{n}\right) \in H \mid h_{1} \geq \cdots \geq h_{n}\right\}$ and $H_{(\leq)}, H_{(>)}, H_{(<)}, H_{(=)}$accordingly.

In order to impose that some points' components are decreasingly ordered, it is common to use permutations. Let $\mathcal{S}_{n}$ be the set of all permutations of $n$ elements, $\sigma \in \mathcal{S}_{n}$ and $\mathbf{x} \in[0,1]^{n}$, we denote by $\mathbf{x}_{\sigma}$ the tuple $\left(x_{\sigma(1)}, \ldots, x_{\sigma(n)}\right)$. Note that for $\mathbf{x}, \mathbf{y} \in \mathbb{R}^{n}$, it holds that $\mathbf{x} \in[0,1]^{n}$ if and only if $\mathbf{x}_{\sigma} \in[0,1]^{n}$. Moreover, for $\mathbf{x}, \mathbf{y} \in[0,1]^{n}$ and $\sigma \in \mathcal{S}_{n}$, it holds that $(\mathbf{x}+\mathbf{y})_{\sigma}=\mathbf{x}_{\sigma}+\mathbf{y}_{\sigma}$, and $\mathbf{x} \cdot \mathbf{y}=\mathbf{x}_{\sigma} \cdot \mathbf{y}_{\sigma}$, where $\mathbf{x} \cdot \mathbf{y}$ denotes the scalar product given by $\mathbf{x} \cdot \mathbf{y}=\sum_{i=1}^{n} x_{i} y_{i}$.

The notion of directional monotonicity, or monotonicity along a ray $\vec{r}$, was introduced in 5], generalizing the notion of monotonicity for functions from $[0,1]^{n}$ to $[0,1]$.

Definition 1. Let $F:[0,1]^{n} \rightarrow[0,1]$ and $\vec{r} \in \mathbb{R}^{n}$, we say that $F$ is $\vec{r}$-increasing (decreasing), if for all $c>0$ and $\mathbf{x} \in[0,1]^{n}$ such that $\mathbf{x}+c \vec{r} \in[0,1]^{n}$, it holds that $F(\mathbf{x}+c \vec{r}) \geq F(\mathbf{x})(F(\mathbf{x}+c \vec{r}) \leq F(\mathbf{x}))$.

Directional monotonicity generalizes weak monotonicity, introduced in [19, which is the particular case of considering as direction the vector $\overrightarrow{1}=(1, \ldots, 1)$.

OD monotonicity was presented in 4. The direction of increasingness of functions that are OD $\vec{r}$-increasing varies in terms of the relative sizes of the input.

Definition 2. Let $F:[0,1]^{n} \rightarrow[0,1]$ and $\vec{r} \in \mathbb{R}^{n}$, we say that $F$ is ordered directionally, $O D, \vec{r}$-increasing (decreasing), if for all $c>0, \sigma \in \mathcal{S}_{n}$ and $\mathbf{x} \in$ $[0,1]^{n}$, it holds that if $\mathbf{x}_{\sigma}, \mathbf{x}_{\sigma}+c \vec{r} \in[0,1]_{(\geq)}^{n}$, then $F\left(\mathbf{x}+c \vec{r}_{\sigma^{-1}}\right) \geq F(\mathbf{x})$ $\left(F\left(\mathbf{x}+c \vec{r}_{\sigma^{-1}}\right) \leq F(\mathbf{x})\right)$. 


\section{The class of strengthened ordered directionally monotone functions}

The concept of SOD monotonicity has been more recently introduced in [11. It is based on OD monotonicity, in fact the difference between both concepts is that SOD monotone functions are not asked to satisfy the condition of comonotonicity between $\mathbf{x}_{\sigma}$ and $\mathbf{x}_{\sigma}+c \vec{r}$.

Definition 3. Let $F:[0,1]^{n} \rightarrow[0,1]$ and $\vec{r} \in \mathbb{R}^{n}$, we say that $F$ is strengthened ordered directionally, SOD, $\vec{r}$-increasing (decreasing), if for all $c>0, \sigma \in \mathcal{S}_{n}$ and $\mathbf{x} \in[0,1]^{n}$, it holds that if $\mathbf{x}_{\sigma} \in[0,1]_{(>)}^{n}$ and $\mathbf{x}_{\sigma}+c \vec{r} \in[0,1]^{n}$, then $F\left(\mathbf{x}+c \vec{r}_{\sigma^{-1}}\right) \geq F(\mathbf{x})\left(F\left(\mathbf{x}+c \vec{r}_{\sigma^{-1}}\right) \leq F(\mathbf{x})\right)$.

If a function is simultaneously (OD, SOD) $\vec{r}$-increasing and (OD, SOD) $\vec{r}$-decreasing, we say that the function is (OD, SOD) $\vec{r}$-constant.

Remark 1. The notation (OD, SOD) $\vec{r}$-increasing refers to directional $(\vec{r}$ increasing), ordered directional (OD $\vec{r}$-increasing) and strengthened ordered directional (SOD $\vec{r}$-increasing) monotonicity.

The case of $\vec{r}=\overrightarrow{0}$ is trivial for the three notions of monotonicity. In fact, every function is (OD, SOD) $\overrightarrow{0}$-constant.

An $\mathrm{OD}$ monotone function $\mathrm{F}$ is required to satisfy the inequality $F(\mathbf{x}+$ $\left.c \vec{r}_{\sigma^{-1}}\right) \geq F(\mathbf{x})$ for points that satisfy the comonotonicity condition $\mathbf{x}_{\sigma}, \mathbf{x}_{\sigma}+$ $c \vec{r} \in[0,1]_{(\geq)}^{n}$, whereas a SOD monotone function $\mathrm{F}$ is required to satisfy the same inequality for points that satisfy that condition and for points that do not. Therefore, SOD $\vec{r}$-increasingness implies OD $\vec{r}$-increasingness. However, the converse statement does not hold.

One of the particularities of each notion of monotonicity is the set of points that satisfy the monotonicity conditions trivially. On the one hand, in the case of directional monotonicity, i.e., $\vec{r}$-increasing functions, the points that trivially satisfy the conditions are those $\mathbf{x} \in[0,1]^{n}$ such that $\mathbf{x}+c \vec{r} \notin[0,1]^{n}$ for all $c>0$. On the other hand, for a function $F$ that is OD (SOD) $\vec{r}$-increasing, such set of points is formed by those $\mathbf{x} \in[0,1]^{n}$ such that if $\sigma \in \mathcal{S}_{n}$ with $\mathbf{x}_{\sigma} \in[0,1]_{(\geq)}^{n}$, then $\mathbf{x}_{\sigma}+c \vec{r} \notin[0,1]_{(\geq)}^{n}\left(\mathbf{x}_{\sigma}+c \vec{r} \notin[0,1]^{n}\right)$ for all $c>0$.

For the case $n=2$, we show in Table 1 the relation of directions (given in terms of the angle that they form with respect to the non-negative horizontal axis) and points that, for each notion of monotonicity, trivially satisfy the conditions.

\section{Properties and connections of the different notions of monotonicity}

We use the following notation to refer to the set of vectors for which a function is increasing (and constant) according to the three different notions of mono- 
Table 1. Directions (in terms of their angle $\alpha$ w.r.t. the non-negative horizontal axis) and points that trivially satisfy the monotonicity conditions for directional, ordered directional and strengthened ordered directional monotonicity

\begin{tabular}{|c|c|c|c|}
\hline $\begin{array}{l}\text { Directions } \\
\vec{r} \text { such that }\end{array}$ & $\begin{array}{c}\text { D monotonicity } \\
\mathbf{x} \in[0,1]^{2} \text { such that }\end{array}$ & $\begin{array}{l}\text { OD monotonicity } \\
\mathbf{x} \in[0,1]^{2} \text { such that }\end{array}$ & $\begin{array}{l}\text { SOD monotonicity } \\
\mathbf{x} \in[0,1]^{2} \text { such that }\end{array}$ \\
\hline$\alpha=0$ & $x_{1}=1$ & \multirow{2}{*}{$x_{1}=1$ or $x_{2}=1$} & \multirow{3}{*}{$x_{1}=1$ or $x_{2}=1$} \\
\hline $0<\alpha \leq \frac{\pi}{4}$ & \multirow[b]{2}{*}{$x_{1}=1$ or $x_{2}=1$} & & \\
\hline$\frac{\pi}{4}<\alpha<\frac{\pi}{2}$ & & $\begin{array}{c}x_{1}=1 \text { or } x_{2}=1 \\
\text { or } x_{1}=x_{2}\end{array}$ & \\
\hline$\alpha=\frac{\pi}{2}$ & $x_{2}=1$ & \multirow{3}{*}{$x_{1}=x_{2}$} & $x_{1}=x_{2}=1$ \\
\hline$\frac{\pi}{2}<\alpha<\pi$ & $x_{1}=0$ or $x_{2}=1$ & & $x_{1}=x_{2}=1$ or $x_{1}=x_{2}=0$ \\
\hline$\alpha=\pi$ & $x_{1}=0$ & & $x_{1}=x_{2}=0$ \\
\hline$\pi<\alpha<\frac{5 \pi}{4}$ & \multirow[t]{2}{*}{$x_{1}=0$ or $x_{2}=0$} & $\begin{array}{c}x_{1}=0 \text { or } x_{2}=0 \\
\text { or } x_{1}=x_{2}\end{array}$ & \multirow{3}{*}{$x_{1}=0$ or $x_{2}=0$} \\
\hline$\frac{5 \pi}{4} \leq \alpha<\frac{3 \pi}{2}$ & & \multirow{2}{*}{$x_{1}=0$ or $x_{2}=0$} & \\
\hline$\alpha=\frac{3 \pi}{2}$ & $x_{2}=0$ & & \\
\hline$\frac{3 \pi}{2}<\alpha<2 \pi$ & $x_{1}=1$ or $x_{2}=0$ & $\begin{array}{c}x_{1}=0 \text { or } x_{1}=1 \\
\text { or } x_{2}=0 \text { or } x_{2}=1\end{array}$ & $\begin{array}{c}x_{1}=0 \text { or } x_{1}=1 \\
\text { or } x_{2}=0 \text { or } x_{2}=1\end{array}$ \\
\hline
\end{tabular}

tonicity with which we deal in this paper. Let $F:[0,1]^{n} \rightarrow[0,1]$, thus we set

$$
\begin{aligned}
\mathcal{D}^{\uparrow}(F) & =\left\{\vec{r} \in \mathbb{R}^{n} \mid F \text { is } \vec{r} \text {-increasing }\right\}, \\
\mathcal{C}(F) & =\left\{\vec{r} \in \mathbb{R}^{n} \mid F \text { is } \vec{r} \text {-constant }\right\},
\end{aligned}
$$

and the remaining sets of directions $\mathcal{D}_{\mathrm{OD}}^{\uparrow}(F), \mathcal{D}_{\mathrm{SOD}}^{\uparrow}(F), \mathcal{C}_{\mathrm{OD}}(F)$ and $\mathcal{C}_{\mathrm{SOD}}(F)$ accordingly.

We derive from the definition the first relation among these sets of directions.

Proposition 1. Let $F:[0,1]^{n} \rightarrow[0,1]$. Then the following items hold:

(i) $\mathcal{C}(F) \subseteq \mathcal{D}^{\uparrow}(F), \mathcal{C}_{O D}(F) \subseteq \mathcal{D}_{O D}^{\uparrow}(F)$ and $\mathcal{C}_{S O D}(F) \subseteq \mathcal{D}_{S O D}^{\uparrow}(F)$;

(ii) $\mathcal{C}_{S O D}(F) \subseteq \mathcal{C}_{O D}(F)$;

(iii) $\mathcal{D}_{S O D}^{\uparrow}(F) \subseteq \mathcal{D}_{O D}^{\uparrow}(F)$.

The following two results are also obtained from the definition of the different notions of monotonicity.

Proposition 2. Let $F:[0,1]^{n} \rightarrow[0,1]$ and $\vec{r} \in \mathbb{R}^{n}$ be such that $r_{1} \geq \ldots \geq r_{n}$. Then $F$ is SOD $\vec{r}$-increasing (decreasing) if and only if $F$ is $O D \vec{r}$-increasing (decreasing). Moreover, $F$ is SOD $\vec{r}$-constant if and only if $F$ is $O D \vec{r}$-constant. 
Proposition 3. Let $F:[0,1]^{n} \rightarrow[0,1]$ be a $(O D, S O D) \vec{r}$-increasing function and let $\varphi:[0,1] \rightarrow[0,1]$ be an increasing (decreasing) function. Then, the composition $\varphi \circ F:[0,1]^{n} \rightarrow[0,1]$ is (OD, SOD) $\vec{r}$-increasing (decreasing).

The vectors' magnitude has no influence whatsoever in the qualification of such vectors as directions of (OD, SOD) increasingness. Therefore, it is possible to limit the set of directions to normalized vectors, i.e., vectors of norm 1 , as it is shown in the next Proposition.

Proposition 4. Let $F:[0,1]^{n} \rightarrow[0,1]$ and $k>0$. Then, $F$ is $(O D, S O D)$ $\vec{r}$-increasing (decreasing) if and only if $F$ is (OD, SOD) $(k \vec{r})$-increasing (decreasing).

The following result reveals a difference between the classes of directionally and ordered directionally monotone functions and the class of strengthened ordered directionally monotone functions.

Proposition 5. Let $F:[0,1]^{n} \rightarrow[0,1] . F$ is $(O D) \vec{r}$-increasing if and only if (OD) $F$ is $(-\vec{r})$-decreasing.

Proof. Case of directional monotonicity:

Let $F$ be $\vec{r}$-increasing and let $\mathbf{x} \in[0,1]^{n}$ and $c>0$ such that $\mathbf{x}-c \vec{r} \in[0,1]^{n}$. Set $\mathbf{y}=\mathbf{x}-c \vec{r}$. Thus,

$$
F(\mathbf{x}+c(-\vec{r}))=F(\mathbf{y}) \leq F(\mathbf{y}+c \vec{r})=F(\mathbf{x}),
$$

hence $F$ is $(-\vec{r})$-decreasing. Similarly, one can show the converse statement.

Case of ordered directional monotonicity:

Let $F$ be OD $\vec{r}$-increasing and let $\mathbf{x} \in[0,1]^{n}, c>0$ and $\sigma \in \mathcal{S}_{n}$ such that $\mathbf{x}_{\sigma}$ and $\mathbf{x}_{\sigma}+c(-\vec{r}) \in[0,1]_{(\geq)}^{n}$. Set $\mathbf{y}=\mathbf{x}+c(-\vec{r})_{\sigma^{-1}}$. Thus, it holds that $\mathbf{y}_{\sigma}=\mathbf{x}_{\sigma}+c(-\vec{r}) \in[0,1]_{(\geq)}^{n}$ and $\mathbf{y}_{\sigma}+c \vec{r}=\mathbf{x}_{\sigma} \in[0,1]_{(\geq)}^{n}$. Now, since $F$ is OD $\vec{r}$-increasing, it holds that

$$
F\left(\mathbf{x}+c(-\vec{r})_{\sigma^{-1}}\right)=F(\mathbf{y}) \leq F\left(\mathbf{y}+c \vec{r}_{\sigma^{-1}}\right)=F(\mathbf{x}),
$$

therefore $F$ is $\mathrm{OD}(-\vec{r})$-decreasing. The converse is analogous.

In 11 it is shown that Proposition 5 does not generally hold for SOD monotonicity, which indicates that whereas the results for (ordered) directional increasingness can be readily extended to (ordered) directional decreasingness, it is not generally the case for the results of SOD monotonicity. This fact is patent in the upcoming results on duality (Section 5).

The next three theorems concern the directions of (OD, SOD) increasingness for functions with some known directions of (OD, SOD) increasingness. The first one, regarding directional monotonicity can be found in [5]; the second, about ordered directional monotonicity, in [4]; and the third, regarding strengthened ordered directional monotonicity, in [11. 
Theorem 1 ([5]). Let $\vec{r}, \vec{s} \in \mathbb{R}^{n}$ and $a, b \geq 0$, with $a+b>0$. Let $\mathbf{x} \in[0,1]^{n}$, $c>0$, and assume that if $\mathbf{x}$ and $\mathbf{x}+c(a \vec{r}+b \vec{s}) \in[0,1]^{n}$, then either $\mathbf{x}+c a \vec{r}$ or $\mathbf{x}+c b \vec{s} \in[0,1]^{n}$. Then, if a function $F:[0,1]^{n} \rightarrow[0,1]$ is both $\vec{r}$-increasing and $\vec{s}$-increasing, then $F$ is also $(a \vec{r}+b \vec{s})$-increasing.

Theorem 2 ([4]). Let $\vec{r}, \vec{s} \in \mathbb{R}^{n}$ and $a, b \geq 0$, with $a+b>0$. Let $\mathbf{x} \in[0,1]^{n}$, $c>0, \sigma \in \mathcal{S}_{n}$ and assume that if $\mathbf{x}_{\sigma}$ and $\mathbf{x}_{\sigma}+c(a \vec{r}+b \vec{s}) \in[0,1]_{(\geq)}^{n}$, then either $\mathbf{x}+c a \vec{r}$ or $\mathbf{x}+c b \vec{s} \in[0,1]_{(\geq)}^{n}$. Then, if a function $F:[0,1]^{n} \rightarrow[0,1]$ is both $O D$ $\vec{r}$-increasing and $O D \vec{s}$-increasing, then $F$ is also $O D(a \vec{r}+b \vec{s})$-increasing.

Theorem 3 ([11]). Let $\vec{r}, \vec{s} \in \mathbb{R}^{n}$ and $a, b \geq 0$, with $a+b>0$. Let $\mathbf{x} \in[0,1]^{n}$, $c>0, \sigma \in \mathcal{S}_{n}$ and assume that if $\mathbf{x}_{\sigma} \in[0,1]_{(\geq)}^{n}$ and $\mathbf{x}_{\sigma}+c(a \vec{r}+b \vec{s}) \in[0,1]^{n}$, then either $\mathbf{x}+c a \vec{r}$ or $\mathbf{x}+c b \vec{s} \in[0,1]^{n}$. Then, if a function $F:[0,1]^{n} \rightarrow[0,1]$ is both SOD $\vec{r}$-increasing and SOD $\vec{s}$-increasing, then F is also SOD $(a \vec{r}+b \vec{s})$ increasing.

Therefore if a function $F$ is (OD, SOD) increasing in two directions $\vec{r}$ and $\vec{s}$, under the assumptions of the preceding theorems, it is also (OD, SOD) increasing in the direction resulting from a positive linear combination of $\vec{r}$ and $\vec{s}$.

\section{Construction methods}

In this Section we show how to construct (ordered, strengthened ordered) directionally monotone functions from functions that have the same type of monotonicity. First, we present some results that establish the relation of a function and its dual according to these generalizations of monotonicity.

Proposition 6. Let $F:[0,1]^{n} \rightarrow[0,1]$ and $F^{c}:[0,1]^{n} \rightarrow[0,1]$ be given by $F^{c}(\mathbf{x})=1-F(\mathbf{x})$. Let $\vec{r} \in \mathbb{R}^{n}$. Then, $F$ is $(O D, S O D) \vec{r}$-increasing if and only if $F^{c}$ is $(O D, S O D) \vec{r}$-decreasing.

Proof. Case of directional monotonicity:

Let $c>0$ and $\mathbf{x}, \mathbf{x}+c \vec{r} \in[0,1]^{n}$, then $F(\mathbf{x}) \leq F(\mathbf{x}+c \vec{r})$ if and only if $F^{c}(\mathbf{x})=1-F(\mathbf{x}) \geq 1-F(\mathbf{x}+c \vec{r})=F^{c}(\mathbf{x}+c \vec{r})$. The cases of OD and SOD monotonicity are straightforward.

As a consequence, the fact that $\left(F^{c}\right)^{c}=F$ yields the following result.

Corollary 1. Let $F:[0,1]^{n} \rightarrow[0,1]$ and $F^{c}:[0,1]^{n} \rightarrow[0,1]$ be given by $F^{c}(\mathbf{x})=$ $1-F(\mathbf{x})$. Let $\vec{r} \in \mathbb{R}^{n}$. Then, $F$ is (OD, SOD) $\vec{r}$-constant if and only if both $F$ and $F^{c}$ are $(O D, S O D) \vec{r}$-increasing. Additionally, the following equalities hold:

(i) $\mathcal{C}(F)=\mathcal{C}\left(F^{c}\right)$;

(ii) $\mathcal{C}_{O D}(F)=\mathcal{C}_{O D}\left(F^{c}\right)$;

(iii) $\mathcal{C}_{S O D}(F)=\mathcal{C}_{S O D}\left(F^{c}\right)$. 
Under the conditions of Proposition 6 , it is clear that $F$ is (OD) $\vec{r}$-increasing if and only if $F^{c}$ is (OD) $(-\vec{r})$-increasing. However, that statement is not generally so for SOD monotonicity.

Proposition 6 and the next result provide the relation of a function and its dual in terms of the directions for which each is (ordered, strengthened ordered) directionally monotone.

Proposition 7. Let $F:[0,1]^{n} \rightarrow[0,1], G:[0,1]^{n} \rightarrow[0,1]$, defined by $G(\mathbf{x})=$ $F(\mathbf{1}-\mathbf{x})$, and $\vec{r} \in \mathbb{R}^{n}$. Let $\vec{r}^{d}=\left(r_{n}, \ldots, r_{1}\right)$. Then

(i) $F$ is $\vec{r}$-increasing if and only if $G$ is $(-\vec{r})$-increasing; and

(ii) $F$ is $O D$ (SOD) $\vec{r}$-increasing if and only if $G$ is OD (SOD) $(-\vec{r})^{d}$ increasing.

Proof. (i) It is straightforward.

(ii) Case of ordered directional monotonicity:

Let $F$ be OD $\vec{r}$-increasing and let $\mathbf{x} \in[0,1]^{n}$. Let $\sigma \in \mathcal{S}_{n}$ and $c>0$ such that $\mathbf{x}_{\sigma} \in[0,1]_{(\geq)}^{n}$ and $\mathbf{x}_{\sigma}+c(-\vec{r})^{d} \in[0,1]_{(\geq)}^{n}$.

Set $\mathbf{y}=\mathbf{1}-\mathbf{x}$ and $\tau \in \mathcal{S}_{n}$ such that $\tau(i)=\sigma(n-i+1)$ for all $i \in\{1, \ldots, n\}$. Thus, one can verify that $\tau^{-1}(i)=n-\sigma^{-1}(i)+1$, and that $\left(\vec{r}^{d}\right)_{\sigma^{-1}}=$ $\vec{r}_{\tau^{-1}}$. Besides, we have that $\mathbf{y}_{\tau} \in[0,1]_{(\geq)}^{n}$ and $\mathbf{y}_{\tau}+c \vec{r} \in[0,1]_{(\geq)}^{n}$. Now, since $F$ is OD $\vec{r}$-increasing, it holds that

$$
G\left(\mathbf{x}+c\left(-\vec{r}^{d}\right)_{\sigma^{-1}}\right)=F\left(\mathbf{y}+c \vec{r}_{\tau^{-1}}\right) \geq F(\mathbf{y})=G(\mathbf{x}),
$$

therefore $G$ is OD $(-\vec{r})^{d}$-increasing.

The converse is straight since $-\left(-\vec{r}^{d}\right)^{d}=\vec{r}$.

Similarly, one can show the case of strengthened ordered directional monotonicity.

Recall that given a function $F:[0,1]^{n} \rightarrow[0,1]$, we can define its dual $F^{d}$ by $F^{d}(\mathbf{x})=1-F(\mathbf{1}-\mathbf{x})$.

Corollary 2. Let $F:[0,1]^{n} \rightarrow[0,1]$ and $F^{d}:[0,1]^{n} \rightarrow[0,1]$ be its dual function. Then, for $\vec{r} \in \mathbb{R}^{n}$, it holds that

(i) $F$ is $\vec{r}$-increasing if and only if $F^{d}$ is $\vec{r}$-increasing;

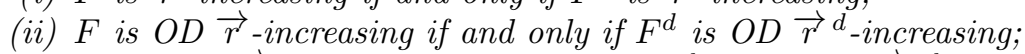

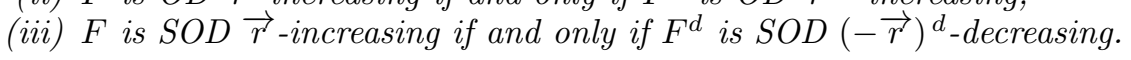

At this point, we expose how a(n) (ordered, strengthened ordered) directionally monotone function can be constructed from a set of $n$ functions with the same type of monotonicity.

Theorem 4. Let $\vec{r} \in \mathbb{R}^{n}$ and $F_{i}:[0,1]^{n} \rightarrow[0,1], 1 \leq i \leq m$, be $m$ (OD, SOD) $\vec{r}$-increasing functions. Let $H:[0,1]^{n} \rightarrow[0,1]$ be an aggregation function. Then the function $H\left(F_{1}, \ldots, F_{m}\right):[0,1]^{n} \rightarrow[0,1]$, given by $H\left(F_{1}, \ldots, F_{m}\right)(\mathbf{x})=$ $H\left(F_{1}(\mathbf{x}), \ldots, F_{m}(\mathbf{x})\right)$, is $(O D, S O D) \vec{r}$-increasing.

In particular, the convex combination of $m$ (OD, SOD) directionally monotone functions is a function with the same kind of monotonicity. 


\section{Characterization of monotonicity in terms of its different generalizations}

The following theorem gives a characterization of the usual condition of increasingness in terms of the different forms of monotonicity discussed in this paper. This result has acquired its present form along with the introduction of the different notions of monotonicity. In [5, one can find the proof of the equivalence of the first two items. the third item was added in [4], and one can find its current presentation in [11.

Theorem 5 ([11]). Let $F:[0,1]^{n} \rightarrow[0,1]$ and $\left(\vec{e}_{1}, \ldots, \vec{e}_{n}\right)$ be the canonical basis of $\mathbb{R}^{n}$. The following are equivalent:

(i) $F$ is increasing;

(ii) $F$ is $\vec{e}_{i}$-increasing for every $i \in\{1, \ldots, n\}$;

(iii) $F$ is $O D \vec{e}_{i}$-increasing for every $i \in\{1, \ldots, n\}$;

(iv) $F$ is $S O D \vec{e}_{i}$-increasing for every $i \in\{1, \ldots, n\}$.

As a consequence, we have another characterization of usual monotonicity, in terms of vectors for which all the components are positive instead of canonical vectors.

Corollary 3. Let $F:[0,1]^{n} \rightarrow[0,1]$. The following are equivalent:

(i) $F$ is increasing;

(ii) $F$ is $O D \vec{r}$ - increasing for every $\vec{r} \in\left(\mathbb{R}^{+}\right)^{n}$;

(iii) $F$ is $S O D \vec{r}$ - increasing for every $\vec{r} \in\left(\mathbb{R}^{+}\right)^{n}$.

Another interesting result is the characterization of weak monotonicity in terms of the other generalizations of monotonicity. Weak monotonicity is a particular case of directional monotonicity which only considers the vector $\overrightarrow{1}=$ $(1, \ldots, 1)$ as a direction.

Theorem 6. Let $F:[0,1]^{n} \rightarrow[0,1]$. The following are equivalent:

(i) F is weakly increasing;

(ii) $F$ is $\overrightarrow{1}$-increasing;

(iii) $F$ is $O D \overrightarrow{1}$-increasing;

(iv) $F$ is $S O D \overrightarrow{1}$-increasing.

Proof. (i) and (ii) are equivalent by the definition of weak monotonicity and Proposition 2 yields that (iii) and (iv) are also equivalent.

Let us show that (ii) and (iii) are equivalent. Assume that $F$ is $\overrightarrow{1}$-increasing and let $\mathbf{x} \in[0,1], \sigma \in \mathcal{S}_{n}$ and $c>0$ such that $\mathbf{x}_{\sigma} \in[0,1]_{(\geq)}^{n}$ and $\mathbf{x}_{\sigma}+c \overrightarrow{1} \in[0,1]_{(\geq)}^{n}$. Since $F$ is $\overrightarrow{1}$-increasing, it holds that $F\left(\mathbf{x}+c \overrightarrow{1}_{\sigma^{-1}}\right)=F(\mathbf{x}+c \overrightarrow{1}) \geq F(\mathbf{x})$. The converse implication is analogous. 


\section{Conclusions}

We have presented the concept of strengthened ordered directional monotonicity, the latest generalization of monotonicity in the literature. We have also discussed some of the main properties and the links among the different stateof-the-art weaker forms of monotonicity, namely, weak monotonicity, directional monotonicity, ordered directional monotonicity and strengthened ordered directional monotonicity. Moreover, we have highlighted some construction methods for classes of functions that are monotone according to the discussed types of monotonicity and we have provided a characterization of the usual condition of monotonicity in terms of directional, ordered directional and strengthened ordered directional monotonicity.

\section{Acknowledgements}

This work is partially supported by the Research Service of Universidad Pública de Navarra and the grants APVV-14-0013 and TIN2016-77356-P (AEI/ FEDER, UE).

\section{References}

1. G. Beliakov, T. Calvo, and T. Wilkin. Three types of monotonicity of averaging functions. Knowledge-Based Systems, 72:114-122, 2014. doi:10.1016/j.knosys. 2014.08 .028

2. G. Beliakov and J. Špirková. Weak monotonicity of Lehmer and Gini means. Fuzzy Sets and Systems, 299:26-40, 2016. doi:10.1016/j.fss.2015.11.006.

3. P. S. Bullen. Handbook of means and their inequalities, volume 560. Springer Science \& Business Media, 2013.

4. H. Bustince, E. Barrenechea, M. Sesma-Sara, J. Lafuente, G. P. Dimuro, R. Mesiar, and A. Kolesárová. Ordered directionally monotone functions. Justification and application. IEEE Transactions on Fuzzy Systems, In press. doi:10.1109/TFUZZ. 2017.2769486.

5. H. Bustince, J. Fernandez, A. Kolesárová, and R. Mesiar. Directional monotonicity of fusion functions. European Journal of Operational Research, 244(1):300-308, 2015. doi:10.1016/j.ejor.2015.01.018

6. T. Calvo, A. Kolesárová, M. Komornıková, and R. Mesiar. A review of aggregation operators. Servicio de Publicaciones de la UAH, Madrid, 2001.

7. G. Choquet. Theory of capacities. Annales de l'institut Fourier, 5:131-295, 1954. doi:10.5802/aif.53

8. L. De Miguel, M. Sesma-Sara, M. Elkano, M. Asiain, and H. Bustince. An algorithm for group decision making using n-dimensional fuzzy sets, admissible orders and OWA operators. Information Fusion, 37:126-131, 2017. doi:10.1016/j.inffus. 2017.01.007.

9. J. García-Lapresta and M. Martínez-Panero. Positional voting rules generated by aggregation functions and the role of duplication. International Journal of Intelligent Systems, 32(9):926-946, 2017. doi:10.1002/int.21877 
10. G. Klir and T. Folger. Fuzzy Sets, Uncertainty, and Information. Prentice Hall, 1988.

11. J. Lafuente, M. Sesma-Sara, A. Roldán, R. Mesiar, and H. Bustince. Strengthened ordered directionally monotone functions. Links between the different notions of monotonicity. Fuzzy Sets and Systems, Submitted for publication.

12. G. Lucca, J. Sanz, G. Dimuro, B. Bedregal, M. J. Asiain, M. Elkano, and H. Bustince. Cc-integrals: Choquet-like copula-based aggregation functions and its application in fuzzy rule-based classification systems. Knowledge-Based Systems, 119:32-43, 2017. doi:10.1016/j.knosys.2016.12.004

13. K. Menger. Statistical metrics. Proceedings of the National Academy of Sciences, 28(12):535-537, 1942.

14. R. Mesiar, A. Kolesárová, and A. Stupňanová. Quo vadis aggregation? International Journal of General Systems, 47(2):97-117, 2018. doi:10.1080/03081079. 2017.1402893.

15. D. Paternain, J. Fernandez, H. Bustince, R. Mesiar, and G. Beliakov. Construction of image reduction operators using averaging aggregation functions. Fuzzy Sets and Systems, 261:87-111, 2015. doi:10.1016/j.fss.2014.03.008

16. M. Sesma-Sara, H. Bustince, E. Barrenechea, J. Lafuente, A. Kolsesárová, and R. Mesiar. Edge detection based on ordered directionally monotone functions. In Advances in Fuzzy Logic and Technology 2017, pages 301-307. Springer, 2017. doi:10.1007/978-3-319-66827-7_27.

17. A. Sklar. Fonctions de répartition à $n$ dimensions et leurs marges. 1959.

18. V. Torra. Hesitant fuzzy sets. International Journal of Intelligent Systems, 25(6):529-539, 2010. doi:10.1002/int.20418

19. T. Wilkin and G. Beliakov. Weakly monotonic averaging functions. International Journal of Intelligent Systems, 30(2):144-169, 2015. doi:10.1002/int.21692

20. R. Yager. On ordered weighted averaging aggregation operators in multicriteria decisionmaking. IEEE Transactions on Systems, Man, and Cybernetics, 18(1):183190, 1988. doi:10.1109/21.87068 